\title{
Method for Analysis of Excitation-Emission Spectral Scans to Characterize Fluorescent Probes in Confocal Microscopy
}

\author{
Vratislav $\mathrm{Cmiel}^{1}$, Josef Skopalik ${ }^{1}$, Larisa Baiazitova ${ }^{1}$ and Ivo Provaznik ${ }^{1,2} *$ \\ 1. Department of Biomedical Engineering, Brno University of Technology, Brno, Czech Republic \\ 2. Department of Physiology, Masaryk University, Brno, Czech Republic. \\ * Corresponding author: provaznik@vut.cz
}

Fluorescent probes, that are designed for various applications, such as measurement of $\mathrm{pH}$, ion concentration within the cytosol of a cell [1], temperature [2], or cell membrane potential [3], show changes in their spectra based on the measured parameter values [4]. The changes in their spectra are often in a form of shift of the excitation and/or emission peak. In addition, the shift may be also influenced by the dye environment (medium).

Spectral properties of the dye are usually represented by 1D excitation-emission spectra of the dye. However, more details of the spectral characteristics can be obtained by excitation-emission 2D scanning [5]. Such analysis is available by the lambda-scan function enabled by Leica TCS SP8X confocal fluorescence microscope, equipped with tunable White Light Laser (WLL) and Hybrid Detectors (HyD). It is technically provided by stepwise shifting the excitation lines of WLL followed by stepwise movement of a narrow detection band in the emission spectrum. Then, the $2 \mathrm{D}$ spectral image representation is calculated. An example of the $2 \mathrm{D}$ spectrum representation obtained by processing of a set of spectral values in a MATLAB environment is shown in Figure 1.

The main aim of the study was to use the spectral excitation-emission 2D scanning followed by 2D spectral analysis in order to obtain an appropriate representation of the properties of the used dye in the original image of a stained cell. We used Di-4-ANNEPS voltage sensitive dye (Invitrogen) binding to an adult rat cardiomyocyte membrane to determine the stability of the dye and to characterize spatial differences of its spectral properties.

In the first phase, we tested influence of several different types of cell environments on the spectral properties of Di-4-ANNEPS dye used for cell staining. Figure 2 shows the position of the excitationemission effective area together with its reduction in case of cell fixation in ethanol (VWR) compared to fixation in formaldehyde (Sigma-Aldrich). The overview of the observed positions of the spectral maximum in different environments is in Table 1.

In the second step, we developed a custom-made tool in MATLAB to process a stack of spectral scans of Di-4-ANNEPS stained cardiomyocytes. Each pixel of the resulted image was colored according to calculated spectral maxima values using a pseudocolor code (see Figure 3). The picture shows spatial and local color differences that may correspond to a binding status or free state of the dye.

A common approach to spectral analysis in living cell experiments is based on 1D spectrum and search for a position of its maximum across whole sample or in selected parts. The described 2D approach to analysis of spectral changes brings new possibilities to analysis of fluorescence probes. It allows analysis in more details and observations of local changes directly from the specimen image. A special impact can be found in the use and analysis of modern hypoxia-hyperoxia sensitive fluorescent particles for precise 
quantification of hypoxia or hyperoxia in microenvironment of studied living cells.

References:

[1] J Han and K. Burgers, Chemical Reviews 110 (2010), p. 2709.

[2] K Okabe, Nature Communications 3 (2012), p. 1.

[3] LB Cohen in "Reviews of Physiology, Biochemistry and Pharmacology 83", (Springer, Berlin, Heidelberg) p. 35.

[4] U Windhorst in "Modern techniques in neuroscience research", eds. U. Windhorst and H. Johansson, (Springer Science \& Business Media, Berlin) p.108.

[5] V Cmiel et al., European Biophysics Journal 46 (2017), p. 433.

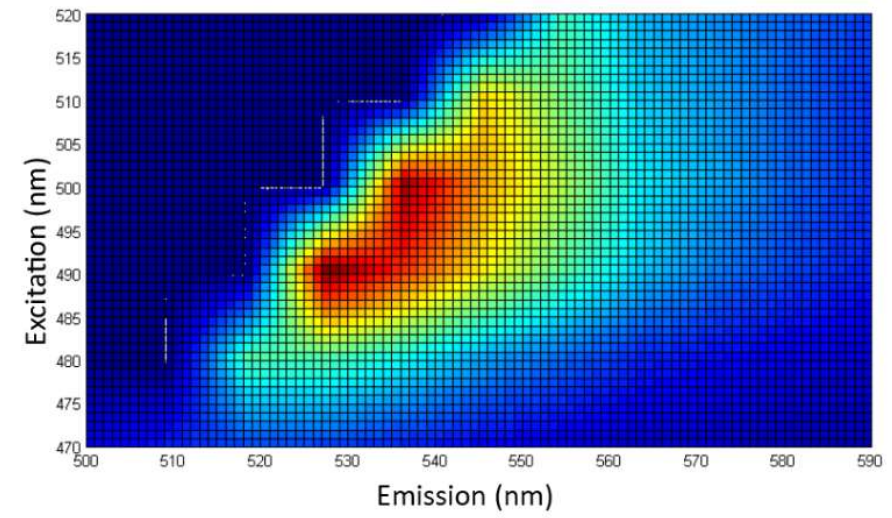

Figure 1. 2D spectral representation obtained by processing of excitation-emission scans.

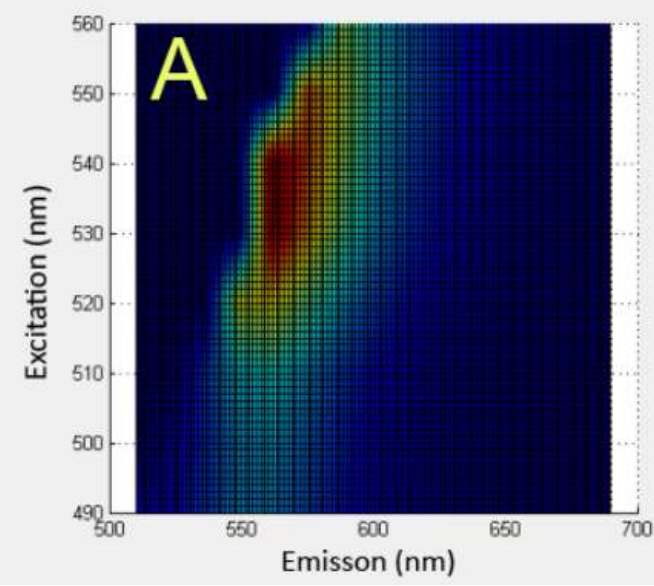

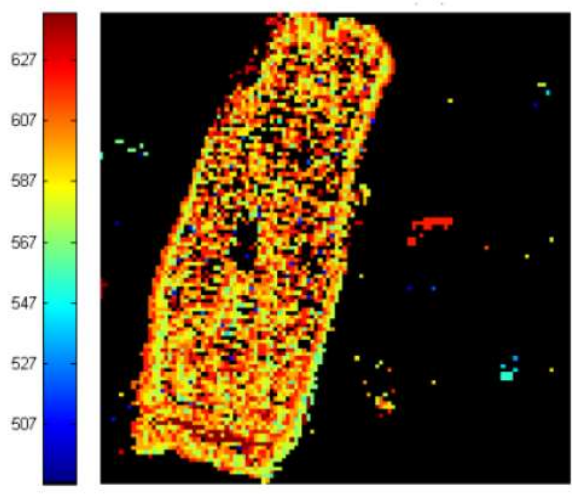

Figure 3. Distribution of emission peak position in cell image. Scale bar represents color codes for emission peak position [nm].

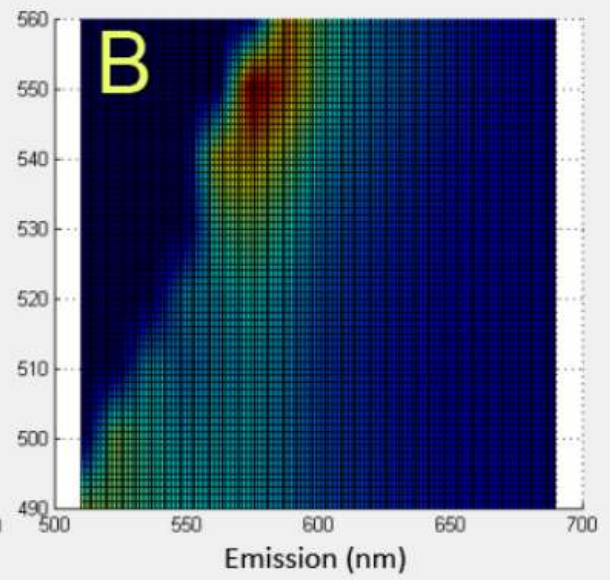

Figure 2. A shift and reduction of excitation-emission effective area for cell fixation in ethanol (A) compared to fixation in formaldehyde (B).

Table 1. Excitation and emission peak positions for various environments. DMEM

\begin{tabular}{|l|c|c|}
\hline \multicolumn{1}{|c|}{ Environment } & Exc. peak [nm] & Emiss. peak [nm] \\
\hline DMEM (Dulbecco's Modified Eagle's Medium) & 560 & 587 \\
\hline DMEM + paraformaldehyde (1:1) & 550 & 574 \\
\hline paraformaldehyde & 530 & 561 \\
\hline paraformaldehyde + ethanol (10:1) & 540 & 561 \\
\hline paraformaldehyde + ethanol (1:1) & 540 & 561 \\
\hline ethanol & 550 & 574 \\
\hline
\end{tabular}

